\title{
Simple strategies versus optimal schedules in multi-agent patrolling ${ }^{* \dagger}$
}

\author{
Akitoshi Kawamura Makoto Soejima
}

\begin{abstract}
Suppose that a set of mobile agents, each with a predefined maximum speed, want to patrol a fence together so as to minimize the longest time interval during which a point on the fence is left unvisited. In 2011, Czyzowicz, Gasieniec, Kosowski and Kranakis studied this problem for the settings where the fence is an interval (a line segment) and a circle, and conjectured that the following simple strategies are always optimal: for Interval Patrolling, the simple strategy partitions the fence into subintervals, one for each agent, and lets each agent move back and forth in the assigned subinterval with its maximum speed; for Circle Patrolling, the simple strategy is to choose a number $r$, place the $r$ fastest agents equidistantly around the circle, and move them at the speed of the $r$ th agent. Surprisingly, these conjectures were then proved false: schedules were found (for some settings of maximum speeds) that slightly outperform the simple strategies.

In this paper, we are interested in the ratio between the performances of optimal schedules and simple strategies. For the two problems, we construct schedules that are 4/3 times (for Interval Patrolling) and 21/20 times (for Circle Patrolling) as good, respectively, as the simple strategies. We also propose a new variant, in which we want to patrol a single point under the constraint that each agent can only visit the point some predefined time after its previous visit. We obtain some similar ratio bounds and NP-hardness results related to this problem.
\end{abstract}

\section{Introduction}

In patrolling problems, a set of mobile agents are deployed in order to protect or supervise a given terrain, and the goal is to leave no point unattended for a long period

${ }^{*}$ Accepted for publication in Theoretical Computer Science [19]. A preliminary version of this paper was announced at the Ninth International Conference on Algorithms and Complexity (CIAC) [18.

${ }^{\dagger}$ This work was supported in part by the Asahi Glass Foundation, JSPS KAKENHI JP17K19960, and a joint research program of NTT and Kyushu University. 
of time. Recent studies [12] have shown that finding an optimal strategy is not at all straightforward, even when the terrain is as simple as it could be. We continue this line of research in three basic settings: patrolling a line segment, a circle, and a point.

\subsection{Interval patrolling}

In 2011, Czyzowicz et al. [8] proposed some simple variants of patrolling problems. One of them was as follow: 1 .

Interval Patrolling Problem. We want to patrol an interval (called the fence) using $k$ mobile agents. We are given their maximum speeds $v_{1}, \ldots, v_{k} \geq 0$ and the idle time $T>0$. For each point $x$ on the fence and time $t \in \mathbb{R}$, there must be an agent who visits $x$ during the interval $[t, t+T)$. How long can the fence be?

Note that if we can patrol a fence of length $L$ with idle time $T$, we can patrol a fence of length $\alpha L$ with idle time $\alpha T$ by scaling the whole schedule by any $\alpha>0$. Thus, we are only interested in the ratio of $L$ and $T$. Unless stated otherwise, we fix the idle time to $T=1$.

Czyzowicz et al. [8] considered the following simple strategy that patrols a fence of length $\left(v_{1}+\cdots+v_{k}\right) / 2$ (with idle time 1 ), and pointed out that no schedule can patrol more than twice as long a fence as this strategy:

Partition-based strategy. Divide the fence into $k$ subintervals of lengths proportional to the maximum speeds of the agents, and let each agent move back and forth on its corresponding subinterval.

They conjectured that this gives the optimal schedule, but it was disproved later: Kawamura and Kobayashi [17] found a setting of maximum speeds $v_{1}, \ldots, v_{k}$ and a schedule that patrols a fence slightly longer than the partition-based strategy does. Thus, a natural question arises: what is the biggest ratio between the performance of an optimal schedule and that of the partition-based strategy? Formally, we want to determine the smallest constant $c$ such that no schedule can patrol a fence of length $c\left(v_{1}+\cdots+v_{k}\right) / 2$.

Czyzowicz et al.'s result [8] mentioned above means that $1 \leq c \leq 2$, and their conjecture was that $c=1$. Kawamura and Kobayashi's example shows that $c \geq 42 / 41$. Later this lower bound was improved to $25 / 24$ [5, 11]. In Section 2.1, we further improve the lower bound to $4 / 3$.

\subsection{Unidirectional circle patrolling}

Czyzowicz et al. [8] also proposed the following (Unidirectional) Circle Patrolling Problem, which we will discuss in Section 3 .

\footnotetext{
${ }^{1}$ In their problem, time is in $[0, \infty)$ instead of $\mathbb{R}$. This difference does not affect our analysis essentially, as we will see at the end of Section 2.2.
} 
Circle Patrolling Problem. We want to patrol a circle using $k$ mobile agents. We are given their maximum speeds $v_{1}, \ldots, v_{k} \geq 0$ and the idle time $T>0$. For each point $x$ on the circle and time $t \in \mathbb{R}$, there must be an agent who visits the point $x$ during the interval $[t, t+T)$. Each agent $i$ can move along the circle clockwise at any speed between 0 and $v_{i}$. How long can the perimeter of the circle be?

Again, we fix the idle time to $T=1$ unless noted otherwise.

Czyzowicz et al. [8] considered the following simple strategy, which patrols a perimeter of length $\max _{r} r v_{r}$ (with idle time 1 ):

Runners strategy. Without loss of generality, we can assume that $v_{1} \geq \cdots \geq v_{k}$. We place the fastest $r$ agents equidistantly on the circle and let them move at speed $v_{r}$, choosing the optimal $r$.

They conjectured that this is optimal, but Dumitrescu et al. [11, Theorem 1] found an example refuting this. Again, we may ask about the ratio of this simple strategy and the optimal schedule. For this problem, we conjecture that the Runners Strategy is not even a constant-ratio approximation strategy. Formally, we suspect that for any constant $c$, there exist $\left(k\right.$ and) $v_{1}, \ldots, v_{k}$ such that we can patrol a perimeter of $c \max _{r} r v_{r}$.

In attempt to progress towards the conjecture, we relate the problem to what we call constant gap families in Section 3.1. Using this relation, we construct a schedule that patrols $1.05 \max _{r} r v_{r}$ in Section 3.2 .

\subsection{Point patrolling}

Consider now a variant of the above circle patrolling problem where there is one special point on the circle, and our goal is to patrol (i.e., visit frequently enough) just this point, rather than all points on the circle. Here, let us assume that a "visit" to the point only happens when an agent arrives at this point (so staying at the point forever does not solve the problem). In this situation, the maximum speed of each agent simply sets a lower bound on the time between its two consecutive visits. This motivates us to introduce the following problem, which we discuss in Section 4 :

Point Patrolling Problem. We want to patrol a point using $k$ agents. We are given minimum gaps $a_{1}, \ldots, a_{k}>0$ and the idle time $T>0$. For each agent $i=1, \ldots, k$, the gaps between two distinct visits by $i$ must always be at least $a_{i}$. Can we patrol the point with idle time $T$ so that, for each time $t \in \mathbb{R}$, there is an agent $i$ that visits the point during the time interval $[t, t+T)$ ?

We could of course consider the problem where we are given $\left(a_{1}, \ldots, a_{k}\right)$ and asked to minimize $T$, but this optimization problem can be reduced to the above decision version by binary search.

As we mention in Section 4.1, this decision problem can be further reduced to a discrete version where the minimum gaps $a_{1}, \ldots, a_{k}$ are positive integers and the goal is to visit the point at each integer time. We study the relation between this problem and the quantity $1 / a_{1}+\cdots+1 / a_{k}$. 
In Section 5, we analyze the complexity of problems that are related to this discretized problem.

\subsection{Related work}

Problems essentially similar to patrolling are discussed under various names and in various real-world contexts, such as maintenance of facilities [2, 3], managing delivery vehicles [4], periodic scheduling [3, 24], and periodic latency problems [6].

While our problems impose frequent visit of every point on the fence (an interval or a circle), some authors consider settings where only parts of the terrain need to be visited often [7]. Other work considers patrolling of more general graphs (rather than just paths and cycles), in which case we may impose frequent visit of vertices [15, 22, 6, 20] or edges [25. Some settings in literature are perhaps motivated by realistic consideration: the patrollers maybe do not see just points but have visibility regions [5, 10]; they may need to stay at a point for a while or walk more slowly when they are looking for intruders rather than just moving [6, 9].

While we are interested in minimizing idle time, i.e., the longest time during which any point is left unvisited, there can be other objectives, such as minimizing the average [13] and using as few agents [4] or as little global knowledge as possible. Some authors consider the intruder as an intelligent player and study the situation in probabilistic or game-theoretic ways [1, 21]. Our setting can be viewed as a special case where we are required to succeed in intercepting the intruder with probability 1.

\section{Interval patrolling}

In the Interval Patrolling Problem (Section 1.1), the fence is an interval $[0, L]$, and a schedule (for agents with maximum speeds $\left.v_{1}, \ldots, v_{k}\right)$ is formally a $k$-tuple $\left(a_{1}, \ldots, a_{k}\right)$ of functions, where each $a_{i}: \mathbb{R} \rightarrow \mathbb{R}$ satisfies $\left|a_{i}(s)-a_{i}(t)\right| \leq v_{i} \cdot|s-t|$ for all $s, t \in \mathbb{R}$. The schedule patrols the fence with idle time $T$ if for each time $t \in \mathbb{R}$ and each location $x \in[0, L]$, there are an agent $i$ and a time $t^{\prime} \in[t, t+T)$ such that $a_{i}\left(t^{\prime}\right)=x$.

In Section 2.1, we prove that for any $c<4 / 3$, there exists a schedule that patrols an interval $c$ times as long as the partition-based strategy. This improves the same claim for $c<25 / 24$ established previously [5, 11].

In Section 2.2, we prove that any schedule can be approximated arbitrarily closely by a periodic schedule. Thus, for any $\varepsilon>0$, we can find in finite time a schedule that is $1-\varepsilon$ times as good as any schedule.

\subsection{A schedule patrolling a long interval}

The goal of this section is the following:

Theorem 1. For any $c<4 / 3$, there are maximum speeds $v_{1}, \ldots, v_{k}$ and a schedule that patrols an interval of length $c\left(v_{1}+\cdots+v_{k}\right) / 2$ (with idle time 1 ). 


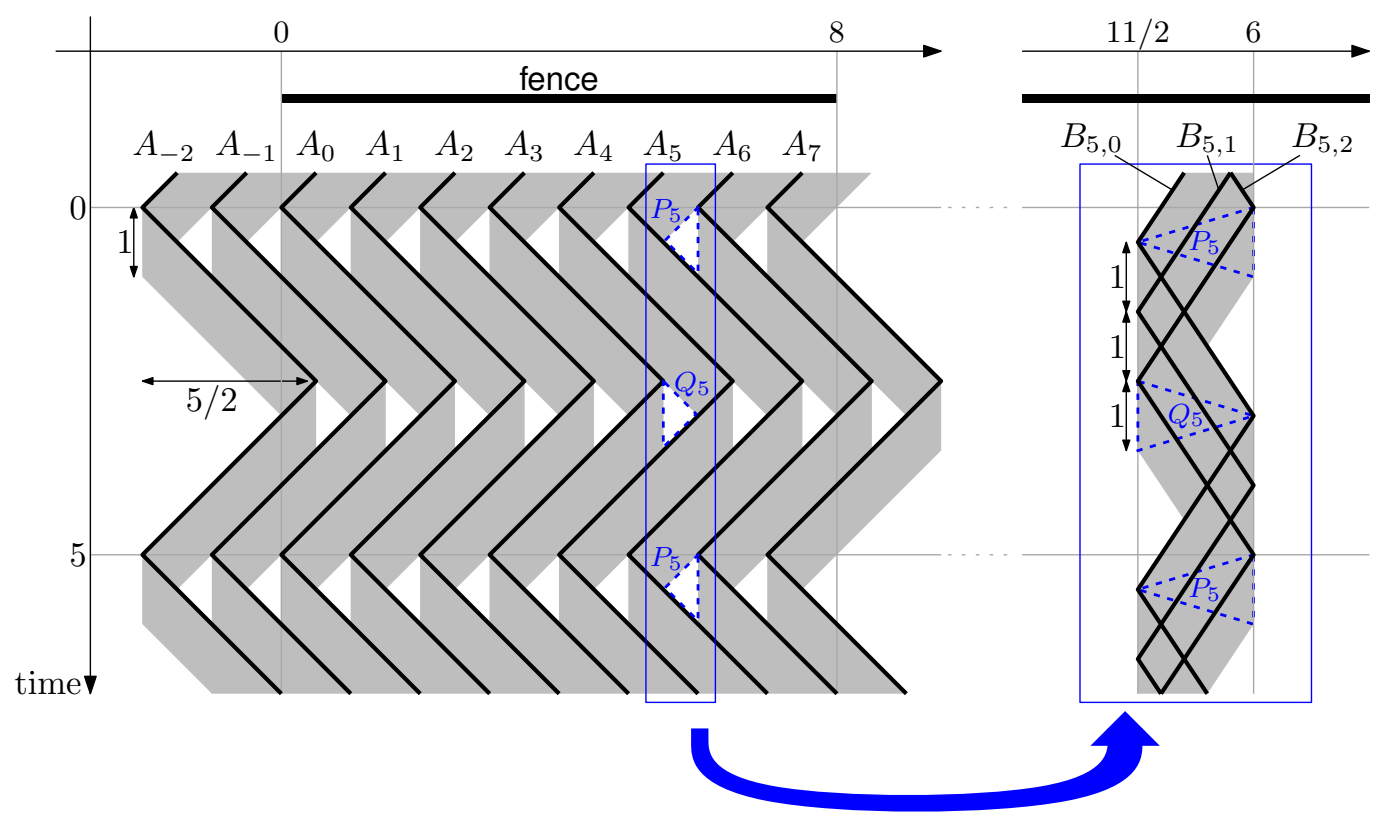

Figure 1: The strategy in the proof of Theorem 1 when $n=3$ and $L=8$. The trajectories of the agents are the thick solid lines, and the regions they cover (the points that have been visited during the past unit time) are shown shaded. The $n+L-1$ faster agents $A_{-n+1}, \ldots, A_{L-1}$ (left) move back and forth with period $2 n-1$, but leave some triangular regions (dotted) uncovered. These regions are covered by the $n L$ slow agents $B_{0,0}, \ldots, B_{n-1, L-1}$ (right; scaled up horizontally for clarity).

Proof. We construct, for each pair of positive integers $n$ and $L$, a schedule that patrols an interval of length $L$ (with idle time 1) using $n+L-1$ agents with maximum speed 1 and $n L$ agents with maximum speed $1 /(2 n-1)$. If the same set of agents follows the partition-based strategy, they would patrol the length $\frac{1}{2}(n+L-1+n L /(2 n-1))$. The ratio between $L$ and this approaches $4 / 3$ when both $n$ and $L / n$ are big, and hence we have the theorem. Our schedule is as follows (Figure 1).

- Each of the $n+L-1$ agents $A_{i}(-n<i<L)$ with speed 1 visits the locations $i$ and $i+n-1 / 2$ alternately (at its maximum speed); it is at location $i$ at time 0 . (This means that some agents occasionally step out of the fence $[0, L]$; to avoid this, we could simply modify the schedule so that they stay at the endpoint for a while.)

- Each of the $n L$ agents $B_{i, j}(0 \leq i<L, 0 \leq j<n)$ with speed $1 /(2 n-1)$ visits the locations $i+1 / 2$ and $i+1$ alternately (at its maximum speed); it is at location $i+1 / 2$ at time $j+1 / 2$.

We claim that this schedule indeed patrols the fence. That is, every $(x, t) \in[0, L] \times \mathbb{R}$ is covered by some agent in the sense that $x$ is visited by this agent during the time interval $[t-1, t]$. 
To see this, note that every agent in this schedule repeats its movement with a period of $2 n-1$. Thus, in this proof, we consider the time modulo $2 n-1$. Also, it is straightforward to verify that the only regions (in the time period $[0,2 n-1]$ ) not covered by the faster agents $A_{i}(-n<i<L)$ are regions $P_{k}$ and $Q_{k}$, for $k=0, \ldots, L-1$, where

- $P_{k}$ is the triangle with vertices $(x, t)=(k+1,0),(k+1,1),(k+1 / 2,1 / 2)$, and

- $Q_{k}$ is the triangle with vertices $(x, t)=(k+1 / 2, n-1 / 2),(k+1 / 2, n+1 / 2),(k+1, n)$

(Figure 1, dotted lines). The region $P_{k}$ is covered by the agents $B_{k, i}$ as they move from $x=k+1$ to $x=k+1 / 2$ during the time interval $[i+n, i+2 n-1 / 2]$. The region $Q_{k}$ is covered by the agents $B_{k, i}$ as they move from $x=k+1 / 2$ to $x=k+1$ during the time interval $[i+1 / 2, i+n]$.

We conjecture $\mathrm{e}^{2}$ that the above construction is optimal:

Conjecture 2. No schedule can patrol an interval that is more than $4 / 3$ times as long as the partition-based strategy.

\subsection{Zigzag schedules}

One of the difficulties about interval (or other) patrolling is that schedules consist of real functions $a_{i}: \mathbb{R} \rightarrow \mathbb{R}$ and we hence cannot check all possible schedules exhaustively. Below, we prove that, for the purpose of discussing bounds on the length of the fence (as we did in Section 2.1), we may restrict attention to a certain class of periodic schedules. Using this, we show that, given the maximum speeds $v_{1}, \ldots, v_{k}>0$ of the agents together with a positive number $\varepsilon>0$, we can find a schedule for them to patrol an interval of length at least $1-\varepsilon$ times the maximum length that can be patrolled.

The movement of an agent during a time interval $\left[t_{\text {start }}, t_{\text {end }}\right] \subseteq \mathbb{R}$ is represented by a function $a:\left[t_{\text {start }}, t_{\text {end }}\right] \rightarrow \mathbb{R}$. This function is called a $(v, \xi)$-zigzag movement, for $v$, $\xi>0$ (Figure 2), if there are integers $p_{0}, p_{1}, p_{2}, p_{3} \in \mathbb{Z}$ such that the agent

- starts at time $t_{\text {start }}$ at location $p_{0} \xi$,

- moves at speed $v$ until it reaches $p_{1} \xi$,

- moves at speed $v$ until it reaches $p_{2} \xi$,

- moves at speed $v$ until it reaches $p_{3} \xi$,

- and then stays there until time $t_{\text {end }}$.

\footnotetext{
${ }^{2}$ While this paper was under review, a disproof of this conjecture was published by Haeupler et al. [16]. They constructed, for each $\varepsilon>0$, an example where the agents can patrol a fence $2(1-\varepsilon)$ times as long as they would with the partition-based strategy.
} 


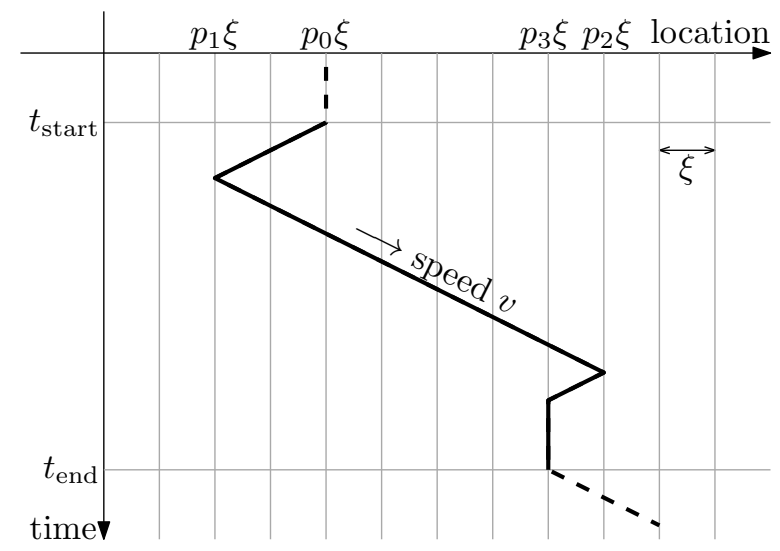

Figure 2: A $(v, \xi)$-zigzag movement on a time interval $\left[t_{\text {start }}, t_{\text {end }}\right]$.

For this movement to be possible, the entire route must be short enough to be traveled with speed $v$; that is,

$$
\left|p_{0}-p_{1}\right| \xi+\left|p_{1}-p_{2}\right| \xi+\left|p_{2}-p_{3}\right| \xi \leq \tau v
$$

where $\tau:=t_{\text {end }}-t_{\text {start }}$ is the length of the time interval.

The following lemma says that any movement of an agent on a time interval can be turned into a zigzag movement taking an only slightly longer time without shrinking the set of points traveled.

Lemma 3. For any positive constants $\delta, \tau, v>0$, we have the following for all sufficiently small $\xi>0$. For any function $a:\left[t_{\text {start }}, t_{\mathrm{end}}\right] \rightarrow \mathbb{R}$ on an interval of length $\tau$ such that $|a(s)-a(t)| \leq v \cdot|s-t|$ for all $s, t \in\left[t_{\text {start }}, t_{\text {end }}\right]$, there is a $(v, \xi)$-zigzag movement $a^{\prime}:\left[(1+\delta) t_{\text {start }},(1+\delta) t_{\text {end }}\right] \rightarrow \mathbb{R}$ such that

- $a^{\prime}\left((1+\delta) t_{\text {start }}\right)=\left\lfloor\frac{a\left(t_{\text {start }}\right)}{\xi}\right\rfloor \xi$ and $a^{\prime}\left((1+\delta) t_{\text {end }}\right)=\left\lfloor\frac{a\left(t_{\text {end }}\right)}{\xi}\right\rfloor \xi$

- any location visited by a is visited by $a^{\prime}$-that is, for each $t \in\left[t_{\mathrm{start}}, t_{\mathrm{end}}\right]$ there is $t^{\prime} \in\left[(1+\delta) t_{\text {start }},(1+\delta) t_{\text {end }}\right]$ such that $a^{\prime}\left(t^{\prime}\right)=a(t)$.

Proof. Let $\xi \leq\left(t_{\text {end }}-t_{\text {start }}\right) v \delta / 5$. Suppose that $a$ takes its minimum and maximum at $t_{\min }, t_{\max } \in\left[t_{\text {start }}, t_{\text {end }}\right]$, respectively. We may assume that $t_{\min } \leq t_{\max }$ (the other case can be treated similarly). Define $a^{\prime}$ to be the $(v, \xi)$-zigzag movement specified by

$$
p_{0}=\left\lfloor\frac{a\left(t_{\text {start }}\right)}{\xi}\right\rfloor, \quad p_{1}=\left\lfloor\frac{a\left(t_{\min }\right)}{\xi}\right\rfloor, \quad p_{2}=\left\lceil\frac{a\left(t_{\max }\right)}{\xi}\right\rceil, \quad p_{3}=\left\lfloor\frac{a\left(t_{\text {end }}\right)}{\xi}\right\rfloor
$$

(see the beginning of Section 2.2 for the meaning of these numbers). This is indeed 
possible: we have (1) for $\tau=(1+\delta)\left(t_{\text {end }}-t_{\text {start }}\right)$ because

$$
\begin{aligned}
& \left(p_{0}-p_{1}\right) \xi+\left(p_{2}-p_{1}\right) \xi+\left(p_{2}-p_{3}\right) \xi \\
& \leq\left(a\left(t_{\text {start }}\right)-a\left(t_{\min }\right)+\xi\right)+\left(a\left(t_{\max }\right)-a\left(t_{\min }\right)+2 \xi\right)+\left(a\left(t_{\max }\right)-a\left(t_{\text {end }}\right)+2 \xi\right) \\
& =\left(a\left(t_{\text {start }}\right)-a\left(t_{\min }\right)\right)+\left(a\left(t_{\max }\right)-a\left(t_{\min }\right)\right)+\left(a\left(t_{\max }\right)-a\left(t_{\text {end }}\right)\right)+5 \xi \\
& \leq\left(t_{\text {min }}-t_{\text {start }}\right) v+\left(t_{\max }-t_{\min }\right) v+\left(t_{\text {end }}-t_{\max }\right) v+5 \xi \\
& =\left(t_{\text {end }}-t_{\text {start }}\right) v+5 \xi \leq(1+\delta)\left(t_{\text {end }}-t_{\text {start }}\right) v .
\end{aligned}
$$

It is straightforward to see that this zigzag movement has the claimed properties.

Next, we prove in the following lemma that any schedule can be converted into one that consists of zigzag movements without deteriorating the idle time too much.

For positive $\xi, \tau>0$, a schedule $\left(a_{1}, \ldots, a_{k}\right)$ (for $k$ agents with maximum speeds $v_{1}$, $\left.\ldots, v_{k}\right)$ is called a $(\xi, \tau)$-zigzag schedule if the movement of each agent $i=1, \ldots, k$ during each time interval $[m \tau,(m+1) \tau], m \in \mathbb{Z}$, is a $\left(v_{i}, \xi\right)$-zigzag movement.

Lemma 4. For any $\varepsilon>0$ and speeds $v_{1}, \ldots, v_{k}>0$, there are $\xi>0$ and $\tau^{\prime}>$ 0 satisfying the following. Suppose that there is a schedule for a set of agents with maximum speeds $v_{1}, \ldots, v_{k}$ that patrols a fence with some idle time $T>0$. Then there is a $\left(\xi, \tau^{\prime}\right)$-zigzag schedule for the same set of agents that patrols the same fence with idle time $T(1+\varepsilon)$.

Proof. We show that it suffices to let $\xi$ be so small that we have the claim of Lemma 3 for

$$
\delta=\frac{\varepsilon}{2}, \quad \tau=\frac{T \delta}{2(1+\delta)}
$$

and for all speeds $v=v_{i}$, and to let $\tau^{\prime}=(1+\delta) \tau$.

Using the schedule $\left(a_{1}, \ldots, a_{k}\right)$ that we start with, we define the claimed $\left(\xi, \tau^{\prime}\right)$-zigzag schedule $\left(a_{1}^{\prime}, \ldots, a_{k}^{\prime}\right)$ as follows. For each agent $i$ and each $m \in \mathbb{Z}$, we define $a_{i}^{\prime}$ on the time interval $\left[m \tau^{\prime},(m+1) \tau^{\prime}\right]$ to be the zigzag movement obtained by Lemma 3 from the movement $a_{i}$ during $[m \tau,(m+1) \tau]$. This defines $a_{i}$ consistently (at multiples of $\tau$ ) because of the first property in Lemma 3 .

To see that this schedule $\left(a_{1}^{\prime}, \ldots, a_{k}^{\prime}\right)$ patrols the fence as claimed, suppose that a location on the fence is left unvisited by the schedule $\left(a_{1}^{\prime}, \ldots, a_{k}^{\prime}\right)$ during a time interval $[\underline{t}, \bar{t}]$ of length $T(1+\varepsilon)$, and hence during its subinterval $\left[\left\lceil\underline{t} / \tau^{\prime}\right\rceil \tau^{\prime},\left\lfloor\bar{t} / \tau^{\prime}\right\rfloor \tau^{\prime}\right]$. By the second property in Lemma 3 , this point is also left unvisited by the schedule $\left(a_{1}, \ldots, a_{k}\right)$ during the time interval $\left[\left\lceil\underline{t} / \tau^{\prime}\right\rceil \tau,\left\lfloor\bar{t} / \tau^{\prime}\right\rfloor \tau\right]$, whose length is

$$
\left(\left\lfloor\frac{\bar{t}}{\tau^{\prime}}\right\rfloor-\left\lceil\frac{\underline{t}}{\tau^{\prime}}\right\rceil\right) \tau \geq\left(\frac{\bar{t}}{\tau^{\prime}}-\frac{\underline{t}}{\tau^{\prime}}-2\right) \tau=\frac{T(1+\varepsilon)}{\tau^{\prime}} \tau-2 \tau=\frac{T(1+2 \delta)}{1+\delta}-2 \tau=T .
$$

The next lemma says that a zigzag schedule can be made periodic without changing the idle time. 
Lemma 5. Suppose that there is a $(\xi, \tau)$-zigzag schedule for a set of agents that patrols a fence with some idle time. Then there is a periodic $(\xi, \tau)$-zigzag schedule for the same agents that patrols the same fence with the same idle time.

Proof. Let $[a, b]$ be the fence, $k$ be the number of agents, and $T$ be the idle time. We may assume that in the given $((\xi, \tau)$-zigzag) schedule, every agent stays within $[A \xi, B \xi]$, where $A=\lfloor a / \xi\rfloor$ and $B=\lceil b / \xi\rceil$, i.e., it never goes far off the fence. In such a schedule, the movement of each agent during each time interval $[m \tau,(m+1) \tau], m \in \mathbb{Z}$, is specified by a quadruple of integers $p_{0}, p_{1}, p_{2}, p_{3} \in\{A, A+1, \ldots, B\}$, and hence there are at most $(B-A+1)^{4}$ possible such movement.

Let $Q=\lceil T / \tau\rceil$. For each $m \in \mathbb{Z}$, there are at most $(B-A+1)^{4 k Q}$ possible ways that the $k$ agents can move during the time interval $[m \tau,(m+Q) \tau]$. Since this is finite, there are integers $m_{0}, m_{1}$ with $m_{0}<m_{1}$ such that in the given $(\xi, \tau)$-zigzag schedule, all agents move during the time interval $\left[m_{1} \tau,\left(m_{1}+Q\right) \tau\right]$ in exactly the same way as they did during $\left[m_{0} \tau,\left(m_{0}+Q\right) \tau\right]$. Consider the periodic schedule, with period $\left(m_{1}-m_{0}\right) \tau$, where each agent perpetually repeats its movement during $\left[m_{0} \tau, m_{1} \tau\right]$ in the original schedule. This schedule patrols the fence with idle time $T$, because the movement of the agents during any time period of length $T$ is identical to their movement in the original schedule in a length- $T$ subinterval of $\left[m_{0} \tau,\left(m_{1}+Q\right) \tau\right]$.

Using the above lemmas, we obtain an algorithm that solves the Interval Patrolling Problem with arbitrarily high precision in the following sense. Suppose that there is a schedule that patrols a fence of length $L>0$ with idle time $T>0$ using agents with maximum speeds $v_{1}, \ldots, v_{k}$. By Lemma 4 , there is a $(\xi, \tau)$-zigzag schedule that patrols a fence of length $(1-\varepsilon) L$, for some $\xi, \tau>0$ determined by the inputs $\varepsilon$ and $v_{1}, \ldots$, $v_{k}$. By Lemma 5, there is a $(\xi, \tau)$-zigzag schedule with period $p$ that patrols the same length $(1-\varepsilon) L$, for some $p>0$ determined by the inputs. Since there are only finitely many $(\xi, \tau)$-zigzag schedules with period $p$, we can check all of them in a finite (though long) time. Thus,

Theorem 6. There is an algorithm that, given $v_{1}, \ldots, v_{k}, T$ and $\varepsilon>0$, finds a schedule that patrols a fence of length at least $1-\varepsilon$ times the length of the fence patrolled by the same agents using any schedule.

In previous work [8, 17, a schedule was defined as functions on the halfline $[0,+\infty)$ (instead of $\mathbb{R}$ ) and the requirement for patrolling was that each location be visited in every length- $T$ time interval contained in this halfline. Note that the argument for Lemmas 4 and 5 in this section stays valid when we start with a patrolling schedule on $[0,+\infty)$. In particular, a patrolling schedule on $[0,+\infty)$ can be converted to a (periodic) schedule on $\mathbb{R}$ without essentially worsening the idle time. Therefore, our slight deviation in the definition does not affect the ratio bounds (discussed in Section 2.1).

\section{Circle patrolling}

As mentioned in Section 1.2, we conjecture that the Runners Strategy for the Circle Patrolling Problem does not have a constant approximation ratio, i.e., that for an arbi- 


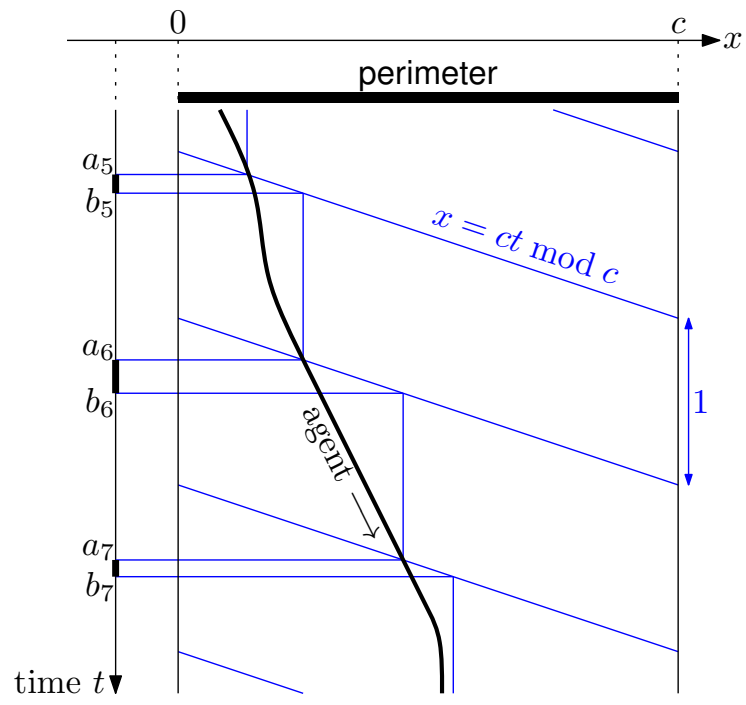

Figure 3: Time intervals $\left[a_{j}, b_{j}\right]$ that are covered by an agent. If the maximum speed of the agent is $v(\leq 1)$, the time $a_{j+1}-a_{j}$ that it takes for it to fall one lap behind the particle (of speed $c$ ) is at most $c /(c-v)$. Thus, $b_{j}-a_{j}=\left(a_{j+1}-1\right)-a_{j} \leq$ $v /(c-v)$.

trarily large $c$, we can find a set of agents with maximum speeds $v_{1}, \ldots, v_{k}$ and their schedule that patrols a perimeter of length $c \max _{r} r v_{r}$. By scaling, we can assume that $\max _{r} r v_{r}=1$. In this case, $v_{i} \leq 1 / i$ for each $i=1, \ldots, k$. Thus, we may and will henceforth assume that $v_{i}=1 / i$.

\subsection{Constant gap families}

In this section, we argue that the above conjecture is equivalent to a perhaps simpler statement about what we call constant gap families below.

Suppose that we want to patrol (with idle time 1) a circle with perimeter $c>1$. By definition, a successful patrolling schedule is one in which, for each time $t \in \mathbb{R}$ and each point $x$ on the circle, some agent visits $x$ during the time interval $[t, t+1]$. Now, instead of imposing this for every $x$, consider the same condition for (each $t$ and) the specific point $x=c t \bmod c$ : we say that an agent covers $t$ if it visits $c t$ mod $c$ during the time interval $[t, t+1]$. In other words, we consider an imaginary particle that moves along the circle at speed $c$, and say that $t$ is covered if the point that the particle passes at time $t$ is then visited by an agent before the particle comes again (Figure 3).

For a schedule to patrol the circle, every time $t \in \mathbb{R}$ must be covered by some agent. This is a necessary, but not a sufficient, condition, because for each point $x$ on the circle, we are requiring a visit only for some of the length-1 time intervals, namely those that start and end when the particle passes $x$. On the other hand, this is a sufficient condition for the schedule to patrol the circle with idle time 2, because every time interval of length 2 contains an interval of length 1 aligned with the particle's visits. Thus, 
Lemma 7. Consider a schedule (of several agents) on a circle of perimeter $c>1$.

1. If the schedule patrols the circle with idle time 1 , every time $t \in \mathbb{R}$ is covered by some agent.

2. If every time $t \in \mathbb{R}$ is covered by some agent, the schedule patrols the circle with idle time 2.

As shown in Figure 3, the set of $t \in \mathbb{R}$ covered by an agent of maximum speed $v<c$ is a union of disjoint intervals $\bigcup_{j \in \mathbb{Z}}\left[a_{j}, b_{j}\right]$ such that

$$
b_{j}-a_{j} \leq \frac{v}{c-v}, \quad a_{j+1}-b_{j}=1
$$

for all $j \in \mathbb{Z}$. Conversely, for any family of intervals $\left(\left[a_{j}, b_{j}\right]\right)_{j \in \mathbb{Z}}$ satisfying $(2)$, an agent with maximum speed $v$ can cover all times in $\bigcup_{j \in \mathbb{Z}}\left[a_{j}, b_{j}\right]$ by a movement in which, for each $j \in \mathbb{Z}$, it is at point $c a_{j} \bmod c$ at time $a_{j}$.

Now, let us assume that the maximum speed of agent $i$ is $1 / i$, as discussed at the beginning of Section 3. Then the set $S_{i} \subseteq \mathbb{R}$ of times covered by agent $i$ is a union of intervals satisfying (2) for $v=1 / i$. That is, it can be written as $S_{i}=\bigcup_{j \in \mathbb{Z}}\left[a_{i, j}, b_{i, j}\right]$ with

$$
b_{i, j}-a_{i, j} \leq \frac{1}{c i-1}, \quad a_{i, j+1}-b_{i, j}=1
$$

for all $j \in \mathbb{Z}$. For a real number $c>1$ and a positive integer $k$, a $(c, k)$-constant gap family (henceforth a $(c, k)$-family) is a $k$-tuple of sets $S_{1}, \ldots, S_{k} \subseteq \mathbb{R}$ satisfying $S_{1} \cup \cdots \cup S_{k}=\mathbb{R}$ and (3). Applying Lemma 7, we have:

Lemma 8. Let $c>1$.

1. If $k$ agents with maximum speeds $1,1 / 2, \ldots, 1 / k$ can patrol a circle of perimeter $c$ (with idle time 1$)$, then there is a $(c, k)$-family.

2. If there is a $(c, k)$-family, then $k$ agents with maximum speeds $1,1 / 2, \ldots, 1 / k$ can patrol a circle of perimeter $c$ with idle time 2 (and thus can patrol a circle of perimeter $c / 2$ with idle time 1$)$.

\subsection{A schedule patrolling a large circle}

As we did for the partition-based strategy in interval patrolling, we may want to ask how good the runners strategy is in comparison to optimal schedules. Our conjecture ${ }^{3}$ is the following:

Conjecture 9. The runners strategy does not have a constant approximation ratio. That is, for any constant $c$, there exist $v_{1}, \ldots, v_{k}$ and a schedule that patrols a circle with perimeter $c \max _{r} r v_{r}$.

\footnotetext{
${ }^{3}$ While this paper was under review, this conjecture was solved affirmatively [16].
} 
By Lemma 8, this holds if and only if for each constant $c$, there are $k$ and a $(c, k)$ family. Note that this is non-trivial already for $c>1$.

We used a computer program and found a $(2.1,122)$-family $\left(S_{1}, \ldots, S_{122}\right)$, which we posted in the ancillary files of the arXiv version of this paper. We were able to finitely describe the whole family, because for each $i \in\{1, \ldots, 122\}$, the set $S_{i}$ has a period of 500 (i.e., for each time $t \in \mathbb{R}$, we have $t \in S_{i}$ if and only if $t+500 \in S_{i}$ ), and the endpoints of all intervals of $S_{i}$ are multiples of $1 / 400$. This also implies that we can check the validity (i.e., $S_{1} \cup \cdots \cup S_{k}=\mathbb{R}$ and the condition (3)) of this example in a straightforward way using a computer program. Thus, by Lemma 8 ,

Theorem 10. There exist $v_{1}, \ldots, v_{k}$ and a schedule that patrols a circle with perimeter $1.05 \max _{r} r v_{r}$.

\section{Point patrolling}

We discuss the Point Patrolling Problem introduced in Section 1.3.

\subsection{Point patrolling in discrete time}

We start by observing that this problem can be reduced to a discretized version:

Discretized Point Patrolling Problem. There are $k$ agents. We are given positive integers $m_{1}, \ldots, m_{k}$. The gap between two distinct visits by agent $i$ must be at least $m_{i}$. Determine whether there is a schedule of visits in which, at each integer time, at least one agent makes a visit.

The list of integers $m=\left(m_{1}, \ldots, m_{k}\right)$ is called good if it admits a patrolling schedule for this problem, and otherwise bad.

Theorem 11. Agents with minimum gaps $a_{1}, \ldots, a_{k}>0$ can patrol the point with idle time $T$ in the (non-discretized) Point Patrolling Problem (see Section 1.3) if and only if $\left(\left\lceil a_{1} / T\right\rceil, \ldots,\left\lceil a_{k} / T\right\rceil\right)$ is good.

Proof. For the 'if' direction, suppose that $\left(\left\lceil a_{1} / T\right\rceil, \ldots,\left\lceil a_{k} / T\right\rceil\right)$ is good. That is, there is a schedule where at each time $j \in \mathbb{Z}$, the point is visited by an agent $i_{j} \in\{1, \ldots, k\}$. Consider the schedule (for the non-discretized problem) where the corresponding visit is made by the same agent $i_{j}$ but now at time $j T$. This schedule is feasible, because the gap between two distinct visits by agent $i$ is now at least $\left\lceil a_{i} / T\right\rceil \cdot T \geq a_{i}$.

Conversely, suppose that the agents achieve the idle time of $T$ in the non-discretized problem. Let $\left(i_{j}, t_{j}\right)_{j \in \mathbb{Z}} \in(\{1, \ldots, k\} \times \mathbb{R})^{\mathbb{Z}}$ be the (ordered) list of all visits in this schedule. That is, the $j$ th visit is made by agent $i_{j}$ at time $t_{j}$, so that

- $0 \leq t_{j+1}-t_{j} \leq T$ for all $j \in \mathbb{Z}$; and

- for all $j, j^{\prime} \in \mathbb{Z}$, if $i_{j}=i_{j^{\prime}}=i$ and $j<j^{\prime}$, then $t_{j^{\prime}}-t_{j} \geq a_{i}$. 
Now consider the schedule in which the $j$ th visit is made by the same agent $i_{j}$ but now at time $j$. This is a feasible schedule for the discretized problem, because, if agent $i$ makes the $j$ th and then the $j^{\prime}$ th visit, then $j^{\prime}-j \geq\left(t_{j^{\prime}}-t_{j}\right) / T \geq a_{i} / T$ by the two conditions above.

Thus, we will henceforth be interested in the Discrete Point Patrolling Problem. We note that this problem can be solved in finite time as follows. Consider a directed graph with $\prod_{i=1}^{k} m_{i}$ vertices, each of which corresponds to a $k$-tuple $\left(b_{1}, \ldots, b_{k}\right)$ with $b_{i} \in\left\{0,1, \ldots, m_{i}-1\right\}$ for each $i$. This vertex means that currently agent $i$ still needs to wait for $b_{i}$ time before its next visit (i.e., time $m_{i}-b_{i}$ has elapsed since its most recent visit). We add an edge from a vertex $\left(b_{1}, \ldots, b_{k}\right)$ to a vertex $\left(b_{1}^{\prime}, \ldots, b_{k}^{\prime}\right)$ when there is $i$ such that $\left(b_{i}, b_{i}^{\prime}\right)=\left(0, m_{i}-1\right)$ and $b_{j}^{\prime}=\max \left\{0, b_{j}-1\right\}$ for each $j \neq i$. A successful patrolling schedule corresponds to an infinite walk in this graph. Thus, $\left(m_{1}, \ldots, m_{k}\right)$ is good if and only if this graph has a cycle.

Using standard algorithms for strongly connected components, we can solve the problem in $O\left(k \prod_{i=1}^{k} m_{i}\right)$ time. We conjecture that this problem is not solvable in polynomial time in general. As we will see in Section 5, similar problems turn out to be NP-complete.

In fact, it is not clear whether this problem is in NP. The above discussion of the directed graph shows that if we can patrol a point with the given agents, then we can do so by a periodic schedule. But it only gives an exponential bound $\prod_{i=1}^{k} m_{i}$ on this period, which is not polynomial in the input length (even if each number $m_{i}$ is given in unary).

\subsection{Quantitative conditions for point patrolling}

For a list $m=\left(m_{1}, \ldots, m_{k}\right)$ of positive integers, define $\operatorname{SumInv}(m)=1 / m_{1}+\cdots+1 / m_{k}$. There is an obvious necessary condition for $a$ to be good:

Theorem 12. If a list $m=\left(m_{1}, \ldots, m_{k}\right)$ of positive integers is good, then SumInv $(m) \geq$ 1.

Proof. As discussed at the end of Section 4.1, there is a periodic patrolling schedule. During each period, each agent $i$ can visit at most $1 / m_{i}$ fraction of the time, so the sum of these fractions over all agents must be $\geq 1$.

The converse does not hold in general (for example, $(2,3,5)$ is bad, see Theorem 17), but it does in a special case:

Lemma 13. If a list $m=\left(m_{1}, \ldots, m_{k}\right)$ of positive integers satisfies $\operatorname{SumInv}(m) \geq 1$, and $m_{i-1}$ divides $m_{i}$ for all $i=2, \ldots, k$, then $m$ is good.

Proof. We prove by induction on $i=1, \ldots, k$ that there is a set $R_{i} \subseteq\left\{1, \ldots, m_{i}\right\}$ of size $\left|R_{i}\right|=m_{i} \cdot \min \left\{\operatorname{SumInv}\left(m_{1}, \ldots, m_{i}\right), 1\right\}$ and a schedule for the first $i$ agents $1, \ldots$, $i$ that visits all times in $m_{i} \mathbb{Z}+R_{i}:=\left\{m_{i} n+r: n \in \mathbb{Z}, r \in R_{i}\right\}$. The lemma then follows from this claim for $i=k$. 
The claim is trivial for $i=1$ (with $R_{1}$ being any set of size 1). Suppose $i>1$. By the assumption, $m_{i}$ is a multiple of $m_{i-1}$, and the induction hypothesis gives a set $R_{i-1} \subseteq\left\{1, \ldots, m_{i-1}\right\}$ of size $m_{i-1} \cdot \min \left\{\operatorname{SumInv}\left(1, \ldots, m_{i-1}\right), 1\right\}$ such that the first $i-1$ agents visit all times in $m_{i-1} \mathbb{Z}+R_{i-1}$. Let $R_{i}$ be the set of numbers in $\left\{1, \ldots, m_{i}\right\}$ congruent modulo $m_{i-1}$ to some element of $R_{i-1}$, so that $\left|R_{i}\right|=\left(m_{i} / m_{i-1}\right) \cdot\left|R_{i-1}\right|$. If $R_{i-1}=\left\{1, \ldots, m_{i-1}\right\}$ (and thus $R_{i}=\left\{1, \ldots, m_{i}\right\}$ and $m_{i-1} \mathbb{Z}+R_{i-1}=m_{i} \mathbb{Z}+R_{i}=\mathbb{Z}$ ), we are done, without even using the additional agent $i$. Otherwise, add any one element $r^{*} \in\left\{1, \ldots, m_{i-1}\right\} \backslash R_{i-1}$ to $R_{i}$, and let agent $i$ visit the times in $m_{i} \mathbb{Z}+\left\{r^{*}\right\}$. Now $\left|R_{i}\right|=m_{i} \cdot \operatorname{SumInv}\left(m_{1}, \ldots, m_{i-1}\right)+1=m_{i} \cdot \operatorname{SumInv}\left(m_{1}, \ldots, m_{i}\right)$.

A corollary to this lemma is the following:

Theorem 14. If a list $m=\left(m_{1}, \ldots, m_{k}\right)$ of positive integers satisfies $\operatorname{SumInv}(m) \geq 2$, then $m$ is good.

Proof. Since the ordering of the agents does not matter, we may assume that $m_{1} \leq$ $\cdots \leq m_{k}$. For each $i=1, \ldots, k$, let $e_{i}$ be the smallest integer with $2^{e_{i}}>m_{i}$. Since $2^{e_{i}} \leq 2 m_{i}$, we have $\operatorname{SumInv}\left(2^{e_{1}}, \ldots, 2^{e_{k}}\right) \geq 1 /\left(2 m_{1}\right)+\cdots+1 /\left(2 m_{k}\right) \geq 1$, and thus we can apply Lemma 13 to conclude that $\left(2^{e_{1}}, \ldots, 2^{e_{k}}\right)$ is good, and hence so is $m$.

In fact, the same argument proves something slightly stronger: if $m_{1}, \ldots, m_{k}$ are positive real numbers with $1 / m_{1}+\cdots+1 / m_{k} \geq 2$, then $m^{\prime}=\left(\left\lfloor m_{1}\right\rfloor+1, \ldots,\left\lfloor m_{k}\right\rfloor+1\right)$ is good. This is obvious when $m_{i}<1$ for some $i=1, \ldots, k$. Otherwise, each $e_{i}$ satisfies $2^{e_{i}} \geq\left\lfloor m_{i}\right\rfloor+1$, and thus the conclusion holds for $m^{\prime}$ instead of $m$.

This (together with Theorem 12) gives a 2-approximation algorithm for the (nondiscretized) Point Patrolling Problem: Given the minimum gaps $a_{1}, \ldots, a_{k}>0$ of agents, let $T$ be the number satisfying $T / a_{1}+\cdots+T / a_{k}=1$. Then $\left(\left\lfloor a_{1} /(2 T)\right\rfloor+1, \ldots\right.$, $\left.\left\lfloor a_{k} /(2 T)\right\rfloor+1\right)$ is good by the aforementioned stronger version of Theorem 14 (with $m_{i}=$ $a_{i} /(2 T)$ ), and hence, by one direction of Theorem 11, we obtain a patrolling schedule with idle time $2 T$. To see that this is indeed a 2-approximation, i.e., that idle time $T^{\prime}<T$ is unachievable, we invoke the other direction of Theorem 11 with $\left(\left\lceil a_{1} / T^{\prime}\right\rceil, \ldots\right.$, $\left.\left\lceil a_{k} / T^{\prime}\right\rceil\right)$, which is bad by Theorem 12 because $\operatorname{SumInv}\left(\left\lceil a_{1} / T^{\prime}\right\rceil, \ldots,\left\lceil a_{k} / T^{\prime}\right\rceil\right)<T / a_{1}+$ $\cdots+T / a_{k}=1$.

For the rest of this section, we will be interested in improving the constant 2 in Theorem 14. The following lemma shows that from a bad list $m$, we can obtain another bad list $m^{\prime}$ whose entries are smaller but whose SumInv is not much smaller:

Lemma 15. Let $Q>0$ and $t \geq 0$ be integers. For a bad list $m$ of positive integers $\leq 2^{t} Q$, there exists a bad list $m^{\prime}$ of positive integers $\leq Q$ such that

$$
\operatorname{SumInv}\left(m^{\prime}\right)+1 \geq\left(1-\frac{2\left(1-2^{-t}\right)}{Q+2}\right) \cdot(\operatorname{SumInv}(m)+1) .
$$

Proof. Once the lemma is proved for $t=1$, we can apply this special statement $t$ times with $Q$ replaced by $2^{i} Q$ for $i=0,1, \ldots, t-1$ to obtain the general statement, because

$$
1-\frac{2\left(1-2^{-t}\right)}{Q+2}=\prod_{i=0}^{t-1}\left(1-\frac{2\left(1-2^{-1}\right)}{2^{i} Q+2}\right)
$$


by a straightforward induction on $t$.

Thus, we shall henceforth assume that $t=1$. Let $r$ and $s$ be the numbers of elements of $m$ that are $\leq Q$ and $>Q$, respectively. Without loss of generality, we can assume that $m$ is in nondecreasing order, so that

$$
m=\left(e_{1}, \ldots, e_{r}, a_{1}, a_{2}, \ldots, a_{s}\right),
$$

where $e_{1} \leq \cdots \leq e_{r} \leq Q<a_{1} \leq a_{2} \leq \cdots \leq a_{s}$. First, define

$$
m^{\prime \prime}=\left(e_{1}, \ldots, e_{r}, a_{2}, a_{2}, a_{4}, a_{4}, \ldots, a_{s-p / 2}, a_{s-p / 2}\right),
$$

where $p \in\{0,1\}$ is the parity of $s$. Thus, $m^{\prime \prime}$ is constructed from $m$ by replacing every other agent with minimum gap $>Q$ by one with the same minimum gap as the next agent, and possibly removing the last agent. Since $m$ is bad, so is $m^{\prime \prime}$. The inverse sum of $m^{\prime \prime}$ can be smaller than that of $m$, but only slightly:

$$
\begin{aligned}
\operatorname{SumInv}(m)-\operatorname{SumInv}\left(m^{\prime \prime}\right) & =\sum_{j=1}^{(s-p) / 2}\left(\frac{1}{a_{2 j-1}}-\frac{1}{a_{2 j}}\right)+p \cdot \frac{1}{a_{s}} \\
& \leq \sum_{i=2}^{s}\left(\frac{1}{a_{i-1}}-\frac{1}{a_{i}}\right)+\frac{1}{a_{s}}=\frac{1}{a_{1}} \leq \frac{1}{Q+1} .
\end{aligned}
$$

Next, define

$$
m^{\prime}=\left(e_{1}, \ldots, e_{r}, b_{1}, \ldots, b_{(s-p) / 2}\right),
$$

where $b_{j}=\left\lceil a_{2 j} / 2\right\rceil$ for $j=1, \ldots,(s-p) / 2$. Two agents with minimum gap $a_{2 j}$ can work as a single agent with minimum gap $b_{j}$, so $\mathrm{m}^{\prime}$ is also bad. Since this rounding $\lceil\cdot\rceil$ increases each element by a factor of $\leq \frac{Q+2}{Q+1}$, we have

$$
\operatorname{SumInv}\left(m^{\prime}\right) \geq \frac{Q+1}{Q+2} \cdot \operatorname{SumInv}\left(m^{\prime \prime}\right) \geq \frac{Q+1}{Q+2}\left(\operatorname{SumInv}(m)-\frac{1}{Q+1}\right),
$$

whence $\operatorname{SumInv}\left(m^{\prime}\right)+1 \geq \frac{Q+1}{Q+2} \cdot(\operatorname{SumInv}(m)+1)$, as was desired.

Theorem 16. If a list $m$ of positive integers satisfies $\operatorname{SumInv}(m)>1.546$, then $m$ is good.

Proof. Suppose that there is a bad $m$ with $\operatorname{SumInv}(m)>1.546$. Using Lemma 15 for $Q=12$ (and a sufficiently large $t$ ), we obtain a bad list $m^{\prime}$ of integers $\leq 12$ such that $\operatorname{SumInv}\left(m^{\prime}\right)+1>(1-2 / 14) \cdot(1.546+1)$, whence SumInv $\left(m^{\prime}\right)>1.1822$. By sorting and truncating $m^{\prime}$ if necessary, we may assume that $m^{\prime}=\left(m_{1}^{\prime}, \ldots, m_{l}^{\prime}\right)$ satisfies

$$
0<m_{1}^{\prime} \leq \cdots \leq m_{l}^{\prime} \leq 12, \quad \frac{1}{m_{1}^{\prime}}+\cdots+\frac{1}{m_{l-1}^{\prime}} \leq 1.1822<\frac{1}{m_{1}^{\prime}}+\cdots+\frac{1}{m_{l}^{\prime}} .
$$

There are only finitely many lists of integers $m^{\prime}$ that satisfy these inequalities. Using a computer program in the arXiv version of this paper, we verified that all of them are good, due to either the sufficient condition in Lemma 13 or the exhaustive searching mentioned at the end of Section 4.1. This is a contradiction. 
The constant in Theorem 16 cannot be made smaller than $\sum_{i=0}^{\infty} 1 /\left(2^{i}+1\right)=1.264 \ldots$ :

Theorem 17. $\left(2,3,5, \ldots, 2^{k}+1\right)$ is bad.

Proof. We prove a stronger claim by induction of $k$ : there is no schedule for these agents (with minimum gaps $2,3,5, \ldots, 2^{k}+1$ ) that visits all of the $2^{k+1}$ consecutive integers, say $1, \ldots, 2^{k+1}$.

When $k=0$, this is trivial. Suppose that the claim holds for $k=t-1$. Assume for the sake of contradiction that there is a schedule for $k=t$ (i.e., for the $t+1$ agents with minimum gaps $\left.2,3,5, \ldots, 2^{t}+1\right)$ that visits $1, \ldots, 2^{t+1}$. Let $p \in\left\{1, \ldots, 2^{t+1}\right\}$ be the first integer that is visited by the agent with minimum gap $2^{t}+1$. Then

- if $p>2^{t}$, the other $t$ agents (with minimum gaps $2,3,5, \ldots, 2^{t-1}+1$ ) must visit integers $1, \ldots, 2^{t}$;

- otherwise, these $t$ agents must visit integers $p+1, \ldots, p+2^{t}$.

In both cases, the induction hypothesis implies that there is no such schedule.

Conjecture 18. If $m$ is a list of positive integers satisfying $\operatorname{SumInv}(m)>\alpha:=$ $\sum_{i=0}^{\infty} 1 /\left(2^{i}+1\right) \approx 1.264$, then $m$ is good.

\section{Complexity of problems related to point patrolling}

We have discussed approximation algorithms for patrolling problems. Finding exactly optimal solutions does not seem to be easy, and in particular, we conjecture that the Discretized Point Patrolling Problem is not pseudo-polynomial-time decidable (i.e., is not polynomial-time decidable even when the input integers $m_{1}, \ldots, m_{k}$ are written in unary). However, we have not been able to show any non-trivial hardness result for this problem. In this section, we prove NP-completeness of two related problems.

\subsection{Disjoint residue classes}

Consider the special case of the Discretized Point Patrolling Problem where the input $m$ satisfies $\operatorname{SumInv}(m)=1$ (see Section 4.2 for SumInv). If $m$ is good in this case, a periodic schedule for it (which exists, as shown at the end of Section 4.1) must use each agent $i$ exactly $1 / m_{i}$ fraction of the time, and hence, the set of times at which $i$ makes a visit must be of the form $m_{i} \mathbb{Z}+r_{i}=\left\{m_{i} n+r_{i}: n \in \mathbb{Z}\right\}$ for some $r_{i} \in \mathbb{Z}$.

Thus, this special case is equivalent to the following problem. For positive integers $m_{1}, \ldots, m_{k}$ and integers $r_{1}, \ldots, r_{k}$, the set $\left\{\left(m_{1}, r_{1}\right), \ldots,\left(m_{k}, r_{k}\right)\right\}$ is called a disjoint covering system [26] if the $k$ sets $m_{1} \mathbb{Z}+r_{1}, \ldots, m_{k} \mathbb{Z}+r_{k}$ are a partition of $\mathbb{Z}$.

Disjoint Covering System Problem. We are given a list $\left(m_{1}, \ldots, m_{k}\right)$ of positive integers. Determine whether there are integers $r_{1}, \ldots, r_{k}$ such that $\left\{\left(m_{1}, r_{1}\right), \ldots,\left(m_{k}, r_{k}\right)\right\}$ is a disjoint covering system. 
Since it is easy to tell whether a given $m=\left(m_{1}, \ldots, m_{k}\right)$ satisfies $\operatorname{SumInv}(m)=1$, this problem is equivalent to the above special case of the Discretized Point Patrolling Problem.

By the Chinese remainder theorem, the sets $m_{i} \mathbb{Z}+r_{i}$ and $m_{j} \mathbb{Z}+r_{j}$ are disjoint if and only if

$$
r_{i} \not \equiv r_{j} \quad\left(\bmod \operatorname{gcd}\left(m_{i}, m_{j}\right)\right),
$$

where $\operatorname{gcd}\left(m_{i}, m_{j}\right)$ is the greatest common divisor of $m_{i}$ and $m_{j}$. Since this condition (4) can be checked easily (for all pairs $(i, j)$ ), the Disjoint Covering System Problem belongs to NP. We conjecture that it is NP-complete:

Conjecture 19. The Disjoint Covering System Problem is NP-complete, even if the inputs $m_{1}, \ldots, m_{k}$ are written in unary.

Failing to prove this, we consider NP-completeness of a similar problem. For positive integers $m_{1}, \ldots, m_{k}$ and integers $r_{1}, \ldots, r_{k}$, the set $\left\{\left(m_{1}, r_{1}\right), \ldots,\left(m_{k}, r_{k}\right)\right\}$ is called a disjoint residue class [23] if the $k$ sets $m_{1} \mathbb{Z}+r_{1}, \ldots, m_{k} \mathbb{Z}+r_{k}$ are pairwise disjoint. Thus, this time the sets need not cover all integers.

Disjoint Residue Class Problem. We are given a list $\left(m_{1}, \ldots, m_{k}\right)$ of positive integers. Determine whether there are integers $r_{1}, \ldots, r_{k}$ such that $\left\{\left(m_{1}, r_{1}\right), \ldots,\left(m_{k}, r_{k}\right)\right\}$ is a disjoint residue class.

Theorem 20. The Disjoint Residue Class Problem is NP-complete, even if the inputs $m_{1}, \ldots, m_{k}$ are written in unary.

Proof. The vertex cover problem for triangle-free graphs is known to be NP-complete. We reduce this problem to the Disjoint Residue Class Problem. Let $G=(V, E)$ be a triangle-free graph with $n=|V|$ vertices and $k=|E|$ edges, and let $s \leq n$ be a positive integer. To the $n$ vertices $u \in V$ of $G$, we assign distinct prime numbers $p_{u}$, all greater than $n$. We rename the edges so that $E=\{1, \ldots, k\}$, and for each edge $i \in E$ with endpoints $u, v \in V$, we let $m_{i}=s p_{u} p_{v}$. We claim that $G$ has a vertex cover of size $s$ if and only if $\left(m_{i}\right)_{i \in E}$ is a yes-instance of the Disjoint Residue Class Problem.

Suppose that $\left\{v_{1}, \ldots, v_{s}\right\} \subseteq V$ is a vertex cover of size $s$. That is, there is a mapping $a: E \rightarrow\{1, \ldots, s\}$ such that each edge $i \in E$ is incident to $v_{a(i)}$. Since less than $n$ edges $i$ are mapped to the same $a(i)$, there is a mapping $b: E \rightarrow\{1, \ldots, n\}$ such that $b(i) \neq b(j)$ for all distinct $i, j \in E$ with $a(i)=b(i)$. For each $i \in E$, let $r_{i}$ be such that $r_{i} \equiv a(i)$ $(\bmod s)$ and $r_{i} \equiv b(i)\left(\bmod p_{v_{a(i)}}\right)$. Then $\left\{\left(m_{i}, r_{i}\right): i \in E\right\}$ is a disjoint residue class, because the condition (4) holds for all distinct $i, j \in E$ as follows:

- If $a(i)=a(j)$ (and hence $b(i) \neq b(j)$ ), then $p_{v_{a(i)}}$ is a common divisor of $m_{i}$ and $m_{j}$, and $r_{i} \equiv b(i) \not \equiv b(j) \equiv r_{j}\left(\bmod p_{v_{a(i)}}\right)$.

- Otherwise, $s$ is a common divisor of $m_{i}$ and $m_{j}$, and $r_{i} \equiv a(i) \not \equiv a(j) \equiv r_{j}(\bmod s)$.

Conversely, let $\left\{\left(m_{i}, r_{i}\right): i \in E\right\}$ be a disjoint residue class. For each $a=1, \ldots, s$, let $E_{a}=\left\{i \in E: r_{i} \equiv a(\bmod s)\right\}$. Then every pair of edges $i, j$ in $E_{a}$ must share a vertex, because otherwise $\operatorname{gcd}\left(m_{i}, m_{j}\right)=s$ and the condition (4) is violated. Since $G$ is triangle-free, there must exist a vertex $v_{a}$ shared by all edges in $E_{a}$. Thus, $\left\{v_{1}, \ldots, v_{s}\right\}$ is a vertex cover. 


\subsection{Patrolling a specified set of times}

We also obtain an NP-complete problem if we specify the set of times at which the point must be visited:

Generalized Point Patrolling Problem. We are given a finite set of times $S \subseteq \mathbb{Z}$ and a list $\left(m_{1}, \ldots, m_{k}\right)$ of positive integers. The gap between two distinct visits by agent $i$ must be at least $m_{i}$. Determine whether there is a schedule of visits in which, at each time in $S$, at least one agent makes a visit.

Theorem 21. The Generalized Point Patrolling Problem is NP-complete, even if the inputs $m_{1}, \ldots, m_{k}$ are written in unary.

Proof. In Numerical 3-Dimensional Matching, we are given three sequences $\left(x_{1}, \ldots, x_{k}\right)$, $\left(y_{1}, \ldots, y_{k}\right),\left(z_{1}, \ldots, z_{k}\right)$ of positive integers written in unary, and we are asked whether there are permutations $\left(p_{1}, \ldots, p_{k}\right)$ and $\left(q_{1}, \ldots, q_{k}\right)$ of $(1, \ldots, k)$ such that

$$
x_{p_{i}}+y_{q_{i}}+z_{i}=b, \quad i=1, \ldots, k,
$$

where $b=\left(x_{1}+\cdots+x_{k}+y_{1}+\cdots+y_{k}+z_{1}+\cdots+z_{k}\right) / k$. We reduce this problem, which is known to be NP-complete [14], to the Generalized Point Patrolling Problem as follows: given the above instance, we construct the input $\left(S, m_{1}, \ldots, m_{k}\right)$ by choosing a sufficiently large number $M$ (say, $\left.M=3 k\left(b+\max \left\{x_{1}, \ldots, x_{k}, y_{1}, \ldots, y_{k}\right\}\right)\right)$ and setting

$$
X_{i}=3 k x_{i}-i, \quad Y_{i}=M-3 k y_{i}+i, \quad m_{i}=M-3 k\left(b-z_{i}\right), \quad i=1, \ldots, k,
$$

and $S=\left\{X_{1}, \ldots, X_{k}, Y_{1}, \ldots, Y_{k}\right\}$. This can clearly be done in polynomial time (even when we need to write $m_{i}$ in unary). Note that for each triple $(p, q, i) \in\{1, \ldots, k\}^{3}$, we have $x_{p}+y_{q}+z_{i} \leq b$ if and only if $Y_{q}-X_{p} \geq m_{i}$.

Thus, if we have (5), there is a patrolling schedule for $\left(S, m_{1}, \ldots, m_{k}\right)$ where each agent $i$ makes visits at $X_{p_{i}}$ and $Y_{q_{i}}$.

Conversely, suppose that there is a patrolling schedule for $\left(S, m_{1}, \ldots, m_{k}\right)$. Since $M$ is (and hence $m_{i}$ are) sufficiently large, no agent can visit more than one of $X_{1}, \ldots, X_{k}$ or more than one of $Y_{1}, \ldots, Y_{k}$. Since the $k$ agents visit the $2 k$ times in $S$, each agent $i$ visits one of $X_{1}, \ldots, X_{k}$ and one of $Y_{1}, \ldots, Y_{k}$, say $X_{p_{i}}$ and $Y_{q_{i}}$. Since $Y_{q_{i}}-X_{p_{i}} \geq m_{i}$, we have $x_{p_{i}}+y_{q_{i}}+z_{i} \leq b$. Since this holds for all $i$, we have (5).

\section{References}

[1] S. Alpern, A. Morton, and K. Papadaki. Patrolling games. Operations Research, 59(5), 1246-1257, 2011.

[2] S. Anily, C. A. Glass, and R. Hassin. The scheduling of maintenance service. Discrete Applied Mathematics, 82(1), 27-42, 1998.

[3] A. Bar-Noy, R. Bhatia, J. Naor, and B. Schieber. Minimizing service and operation costs of periodic scheduling. Mathematics of Operations Research, 27(3), 518-544, 2002 . 
[4] A. M. Campbell and J.R. Hardin. Vehicle minimization for periodic deliveries. European Journal of Operational Research, 165(3), 668-684, 2005.

[5] K. Chen, A. Dumitrescu, and A. Ghosh. On fence patrolling by mobile agents. In Proc. 25th Canadian Conference on Computational Geometry (CCCG), 2013.

[6] S. Coene, F. C.R. Spieksma, and G. J. Woeginger. Charlemagne's challenge: the periodic latency problem. Operations Research, 59(3), 674-683, 2011.

[7] A. Collins, J. Czyzowicz, L. Gąsieniec, A. Kosowski, E. Kranakis, D. Krizanc, R. Martin, and O. Morales Ponce. Optimal patrolling of fragmented boundaries. In Proc. 25th ACM Symposium on Parallelism in Algorithms and Architectures (SPAA), 241-250, 2013.

[8] J. Czyzowicz, L. Gąsieniec, A. Kosowski, and E. Kranakis. Boundary patrolling by mobile agents with distinct maximal speeds. In Proc. 19th European Symposium on Algorithms (ESA), LNCS 6942, 701-712, 2011.

[9] J. Czyzowicz, K. Georgiou, E. Kranakis, F. MacQuarrie, and D. Pajak. Fence patrolling with two-speed robots. In Proc. Fifth International Conference on Operations Research and Enterprise Systems (ICORES), pp. 229-241, 2016.

[10] J. Czyzowicz, E. Kranakis, D. Pajak, and N. Taleb. Patrolling by robots equipped with visibility. In Proc. 21st International Colloquium on Structural Information and Communication Complexity (SIROCCO), LNCS 8576, 224-234, 2014.

[11] A. Dumitrescu, A. Ghosh, and C. D. Tóth. On fence patrolling by mobile agents. Electronic Journal of Combinatorics, 21, P3.4, 2014.

[12] A. Dumitrescu and C.D. Tóth. Computational Geometry Column 59. ACM SIGACT News, 45(2), 68-72, 2014.

[13] Y. Elmaliach, A. Shiloni, and G. A. Kaminka. A realistic model of frequency-based multi-robot polyline patrolling. In Proc. Seventh International Joint Conference on Autonomous Agents and Multiagent Systems (AAMAS), 63-70, 2008.

[14] M. Garey and D. S. Johnson. Computers and Intractability: A Guide to the Theory of NP-Completeness. W.H. Freeman, 1979.

[15] B. Gorain and P. S. Mandal. Approximation algorithms for sweep coverage in wireless sensor networks. Journal of Parallel and Distributed Computing, 74, 2699-2707, 2014 .

[16] B. Haeupler, F. Kuhn, A. Martinsson, K. Petrova, and P. Pfister. Optimal strategies for patrolling fences. In Proc. 46th International Colloquium on Automata, Languages, and Programming (ICALP), LIPIcs 132, Article 144, 2019. 
[17] A. Kawamura and Y. Kobayashi. Fence patrolling by mobile agents with distinct speeds. Distributed Computing, 28(2), 147-154, 2015.

[18] A. Kawamura and M. Soejima. Simple strategies versus optimal schedules in multiagent patrolling. In Proc. Ninth International Conference on Algorithms and Complexity (CIAC), LNCS 9079, 261-273, 2015.

[19] A. Kawamura and M. Soejima. Simple strategies versus optimal schedules in multiagent patrolling. Theoretical Computer Science, 839, 195-206, 2020.

[20] 能城秀涁．複数の巡査の協力による指定地点の警邏について．東京大学総合文 化研究科修士論文，平成 30 年. H. Noshiro. Collaborative patrolling of designated points on graphs. Master Thesis, Graduate School of Arts and Sciences, University of Tokyo, 2018. In Japanese.

[21] K. Papadaki, S. Alpern, T. Lidbetter, and A. Morton. Patrolling a border. Operations Research, 64(6), 1256-1269, 2016.

[22] F. Pasqualetti, A. Franchi, and F. Bullo. On cooperative patrolling: optimal trajectories, complexity analysis, and approximation algorithms. IEEE Transactions on Robotics, 28(3), 592-606, 2012.

[23] Z. Sun. On disjoint residue classes. Discrete Mathematics, 104(3), 321-326, 1992.

[24] J. Sgall, H. Shachnai, and T. Tamir. Periodic scheduling with obligatory vacations. Theoretical Computer Science, 410, 5112-5121, 2009.

[25] V. Yanovski, I. A. Wagner, and A. M. Bruckstein. A distributed ant algorithm for efficiently patrolling a network. Algorithmica, 37, 165-186, 2003.

[26] Š. Znám. On exactly covering systems of arithmetic sequences. Mathematische Annalen, 180(3), 227-232, 1969. 